\title{
Avaliação dos problemas enfrentados no manejo do caramujo gigante africano Achatina fulica (Gastropoda: Pulmonata) no Brasil
}

\author{
Eduardo Colley ${ }^{1,2}$ \& Marta Luciane Fischer ${ }^{2}$
}

\begin{abstract}
1 Programa de Pós-Graduaçao em Zoologia, Departamento de Zoologia, Universidade Federal do Paraná. Centro Politécnico, Caixa Postal 19020, 81531-990 Curitiba, Paraná, Brasil. E-mail: eduardocolley@yahoo.com.br

2 Núcleo de Estudos do Comportamento Animal, Departamento de Biologia, Pontifícia Universidade Católica do Paraná. Avenida Silva Jardim 1664/1101, 80250200 Curitiba, Paraná, Brasil. E-mail: marta.fischer@pucpr.br
\end{abstract}

\begin{abstract}
Evaluation of the problems faced in the management of the giant African snail Achatina fulica (Gastropoda: Pulmonata) in Brazil. The giant African snail Achatina fulica Bowdich, 1822 was introduced into Paraná, Brazil, in the 1980s. Since then, it has spread across the country, despite its known invasive status on a global scale. The main objective of this study was to assess the problems faced in the management of the giant African snail. To accomplish that, we gathered and analyzed information available on the internet, scientific databases, field studies, as well as interviews and consultations with official agencies, NGOs, museums, the scientific community and people that coexist with the species. Despite the fact that extensive information has been generated regarding $A$. fulica (1,340 articles, 65 summaries in national annals, 40,700 pages on the internet), essential to promote control measures to hinder the propagation of this species, the majority of the available information remains inaccessible to the world at large. Moreover, the environmental, economic and health impacts of this species remain unclear, which may contribute to discouraging the initiation of management actions. Finally, control measures are still inefficient due to the generalist profile of this species and the lack of knowledge concerning its biology and ecology. Thus, in conclusion, even though A. fulica has been an invasive species for over a century in many other countries, it is difficult to find evidence supporting the need to subsidize its management. This may be an indication that many other, poorly known exotic species will have time and the proper conditions to become established and cause problems before they come to be recognized as invasive.
\end{abstract}

KEY WORDS. Biological invasions; control; economic; environmental; impacts; invasive exotic species; health.

As invasões biológicas são consideradas a segunda causa da perda de biodiversidade, pois podem alterar os ciclos ecológicos e homogeneizar a biota (Byers et al. 2002). O impacto causado pelas espécies invasoras evidencia a ineficiência na prevenção das invasões, bem como na detecção e no controle dos casos diagnosticados. Apesar dos vários estudos sobre invasões biológicas, a falta de diretrizes para elaboração de programas de manejo, que conciliem a teoria ecológica e a prática conservacionista, tem impedido a efetivação das ações de manejo (Byers et al. 2002).

No Brasil, um caso de invasão atual e comum a muitos municípios do país, é o caramujo gigante africano Achatina fulica Bowdich, 1822. O molusco é considerado uma das cem piores espécies exóticas invasoras do planeta (Lowe et al. 2004), uma vez que a sua rusticidade e os seus hábitos generalistas, aliados ao elevado potencial reprodutivo e ao grande porte dos adultos, favorecem a adaptação e a colonização de diferentes ambientes. Nas áreas onde foi introduzido, se tornou um sério problema ambiental e econômico, devido à competição por alimento e espaço com a fauna nativa e à destruição de cultivos agrícolas (Teles \& FonTes 2002). A espécie também é considerada um pro- blema de saúde pública, devido à potencialidade de ser hospedeira de nematóides causadores da angiostrongilíase humana (Teles \& Fontes 2002). A dispersão passiva, intencional ou acidental, é o principal meio de disseminação do caramujo.

No Brasil, o caramujo africano foi introduzido no final da década de 1980, como alternativa para criação comercial e consumo humano. Atualmente, ocorre em populações elevadas na área urbana de vários municípios de 23 estados (Teles \& FonTES 2002, THIENGo et al. 2007), gerando preocupação de órgãos governamentais e não governamentais. Um protocolo para o diagnóstico e monitoramento das populações vem sendo elaborado (Fischer \& Colley 2004, 2005, Simião \& FisCher 2004, FisCher et al. 2006) e visa subsidiar e direcionar os planos de manejo.

As informações disponíveis sobre $A$. fulica servem como subsídio de conhecimento sobre a espécie permitindo estabelecer os parâmetros que evidenciam quando o caramujo representa uma população estabelecida, caracterizando-o como espécie exótica invasora. Os dados sobre sua biologia e dinâmica populacional permitem determinar os elementos que podem prevenir que o molusco se torne invasor, contribuindo para 
estabelecer diretrizes sobre análise de risco, detecção precoce e manejo. Entretanto, apesar destas informações estarem disponíveis, o que tem ocorrido em inúmeros países é uma invasão cada vez maior por A. fulica. Logo, hipotetiza-se que o insucesso no manejo do caramujo africano, bem como a dificuldade no seu estabelecimento, se deve à inexistência de informações acessíveis com relação: aos conhecimentos científicos; às experiências bem ou mal sucedidas de controle; aos reais impactos econômicos, ambientais e na saúde pública; e à legislação. Assim, o presente estudo teve como objetivo avaliar as dificuldades enfrentadas no manejo de $A$. fulica no país.

\section{MATERIAL E MÉTODOS}

O presente estudo foi realizado entre 2003 e 2007 e desenvolvido em três etapas: análise bibliográfica; entrevistas com comunidade científica, sociedade e órgãos governamentais e não governamentais; e pesquisa experimental em campo.

A qualificação e quantificação da literatura disponível sobre $A$. fulica foi realizada através da consulta às bases de indexação eletrônicas e impressas do Biological Abstracts, Zoological Records, CAB database (Commonwealth Agricultural Bureaux), EBSCO (base de dados bibliográficos em textos completos), Google Scholar e referências bibliográficas de artigos e livros científicos. As revistas científicas disponíveis no Brasil foram localizadas através do buscador eletrônico IBICT (http:/ /www.ibict.br/), para verificar a acessibilidade do periódico em nível nacional e local. A consulta à literatura disponível também incluiu os resumos de anais de congressos brasileiros de 1995 a 2005 (Congressos Brasileiros de Zoologia, Encontro Anual de Etologia e Encontro Brasileiro de Malacologia) e toda a informação veiculada sobre $A$. fulica no sistema de informação global da rede mundial de computadores interconectados (Internet) a partir do site eletrônico do Google (http:// www.google.com.br) utilizando como objeto de busca o binômio "Achatina fulica". O assunto reportado nos trabalhos obtidos foi avaliado quanto: 1) ao tema abordado: criação comercial, impacto, manejo, história natural (sistemática, biologia, ecologia), fisiologia (neurologia, bioquímica, genética e fisiologia), legislação, manejo e outros; 2) à origem da informação: governamental, universidades, ONGs, empresas, mídia e páginas pessoais; 3) ao tipo de informação: científica, popular, incentivo à criação, divulgação e técnica; e 4) ao ano da publicação. A análise do impacto causado pela invasão de $A$. fulica foi realizada sob os aspectos econômicos, saúde humana e ambiental. Esta avaliação se deu a partir de entrevistas semiestruturadas com 44 pesquisadores que atuam com malacologia no Brasil e no exterior ou desenvolvem pesquisas na Floresta Atlântica no estado do Paraná e com 30 residentes abordados aleatoriamente dos municípios de Morretes e 30 de Guaraqueçaba, ambos localizados no litoral do estado do Paraná, onde a ocorrência do caramujo foi previamente confirmada (Fischer \& Colley 2004, Fischer et al. 2006). Ambos os grupos foram questionados quanto ao tipo de impacto (econô- mico, saúde humana e ambiental) que a introdução de $A$. fulica tem gerado.

A análise do impacto econômico ainda envolveu consulta aos órgãos gestores da agricultura no Paraná. A avaliação do impacto sobre a saúde humana contou com um levantamento de dados junto à Faculdade de Biociências/Pesquisas Biomédicas (PUCRS - Biociências), Fundação Oswaldo Cruz (Fiocruz/RJ) e Secretaria de Estado da Saúde do Paraná (SESA/PR).

O impacto ambiental também foi avaliado no Paraná pela comparação de populações de $A$. fulica e de caramujos nativos de duas outras famílias (Megalobulimidae e Strophocheilidae) que apresentam porte similar ao de $A$. fulica e poderiam estar sofrendo efeitos negativos da competição estabelecida pelo invasor. Em campo, no município de Guaraqueçaba, Paraná, foi realizada uma avaliação comparativa entre o tamanho das populações da espécie invasora e dos caramujos nativos durante o verão de 2005. Para tanto, foram realizadas coletas manuais e a quantificação de todas as conchas e exemplares de A. fulica, Megalobulimidae e Strophocheilidae ocorrentes em 59 terrenos da área urbana. As coletas e a caracterização das populações foram realizadas de acordo com Fischer \& Colley $(2004,2005)$. Foi relizada, também, uma consulta qualitativa às Coleções Malacológicas do Museu de História Natural do Capão da Imbuia (MHNCI, Curitiba) e do Museu de Zoologia da Universidade de São Paulo (MZUSP, São Paulo), sendo registrados os locais de ocorrência e quantidade de $A$. fulica e dos gastrópodes nativos das duas famílias adicionais tombados nestas instituições.

O potencial de dispersão passiva e ativa de $A$. fulica, foi avaliado tendo como hipótese que esses animais são constantemente re-introduzidos nas comunidades através do transporte passivo em material de construção e através do deslocamento pelo ambiente, fato que inviabiliza o controle pontual feito pelo morador. O mecanismo de dispersão passiva foi verificado através de vistorias em duas empresas distribuidoras de material de construção localizadas no município de Paranaguá e duas em Guaraqueçaba, ambos localizados no litoral do Paraná.

A avaliação do potencial de recolonização de $A$. fulica foi realizada em Guaraqueçaba, onde havia sido confirmada a presença abundante do caramujo invasor nos jardins da maioria das residências (Fischer \& Colley 2004, 2005). Para tanto, foram conduzidas duas coletas diurnas, uma durante o verão e outra no inverno de 2005. Nas duas campanhas foram coletados e mensurados todos os caramujos encontrados em 11 terrenos sorteados a partir do mapa da cidade, sendo vistoriados os mesmos terrenos nas duas estações do ano. Também foi realizada a caracterização da população de $A$. fulica de acordo com o protocolo utilizado por Fischer \& Colley (2004, 2005). Para esta análise não foi considerado se os terrenos vistoriados apresentavam o entorno invadido por $A$. fulica.

Os programas de manejo e a legislação sobre espécies invasoras foram analisados pela consulta aos órgãos gestores: Ministério do Meio Ambiente (MMA), Instituto Brasileiro do Meio Ambiente e Recursos Naturais e Renováveis (Ibama) e 
SESA/PR. A análise foi realizada quanto à data de promulgação das leis, termos e definições, início e duração das ações, forma de manejo utilizada, objetivos, abrangência e aplicação tanto das leis quanto das ações de manejo.

Para as análises dos dados de frequência (tipo de literatura e taxa de re-colonização) foi utilizado o teste qui-quadrado composto, considerando como hipótese nula que as opções analisadas ocorressem ao acaso e apresentassem a mesma frequência de ocorrência. Para análise das médias cujos dados não possuíam distribuição normal (população do invasor e das espécies nativas e taxa de re-colonização) foi utilizado o teste Mann-Whitney (U).

O presente projeto foi executado segundo as legislações brasileiras vigentes, possuindo autorização de coleta, transporte e manutenção de animais emitida pelo Ibama/PR (02017003886/2003-41) e aprovação por parte do Comitê de Ética em uso de Animais (CEUA-PUCPR protocolo 67) e Comitê de Ética em Pesquisa com Humanos (CEP-PUCPR 515). Todos os entrevistados estavam cientes da finalidade do questionário e concordaram com a participação na pesquisa, assinando o termo de livre consentimento sobre utilização das respostas concedidas nos resultados do presente estudo, os quais estão arquivados no Laboratório Núcleo de Estudos do Comportamento Animal, Pontifícia Universidade Católica do Paraná (PUCPR). Espécimes testemunho foram depositados na coleção científica do Museu de Zoologia, PUCPR sob o número de tombo 11611.

\section{RESULTADOS}

\section{Qualificação e quantificação da literatura disponível}

O inventário da bibliografia produzida a respeito de $A$. fulica resultou em 1340 registros, sendo principalmente na área da fisiologia $(32,3 \%)$ e da história natural $(25,7 \%)\left(\chi_{(6)}^{2}=609\right.$; $\mathrm{p}<0,01)$. Ao longo das últimas 11 décadas houve aumento da produção bibliográfica, porém os temas predominantes em cada década variaram, sendo que a sistemática dominou nos primeiros artigos, o controle e história natural na década de 1960 e 1970 e os estudos fisiológicos no final do século XX (Fig. 1). Apesar da existência de informações relativas à espécie, apenas $35 \%$ das revistas científicas estavam disponíveis nas bibliotecas brasileiras, sendo que destas, 50\% estavam em bibliotecas do estado de São Paulo e 24\% no Paraná.

O levantamento das informações sobre $A$. fulica disponíveis na internet em 2005, resultou em aproximadamente 40.700 páginas, enquanto em 2007 este número praticamente duplicou para 87.500. No entanto, em ambos os anos somente as 780 primeiras páginas estavam disponíveis para serem acessadas, devido à estrutura do site de busca. A análise dos sites revelou predomínio de informações científicas $(42,7 \%)$ e de divulgação $(31,8 \%)$ em comparação com as populares $(18 \%)$, técnicas $(5,5 \%)$ e de estímulo à criação $(1,7 \%)\left(\chi_{(4)}^{2}=380,5 ; \mathrm{p}<0,01\right)$, tendo como origem significativamente mais entidades acadêmicas $(44,8 \%$ - revistas científicas, artigos, anais de congressos e museus) e governamentais $(18,8 \%$ - leis, decretos, instruções

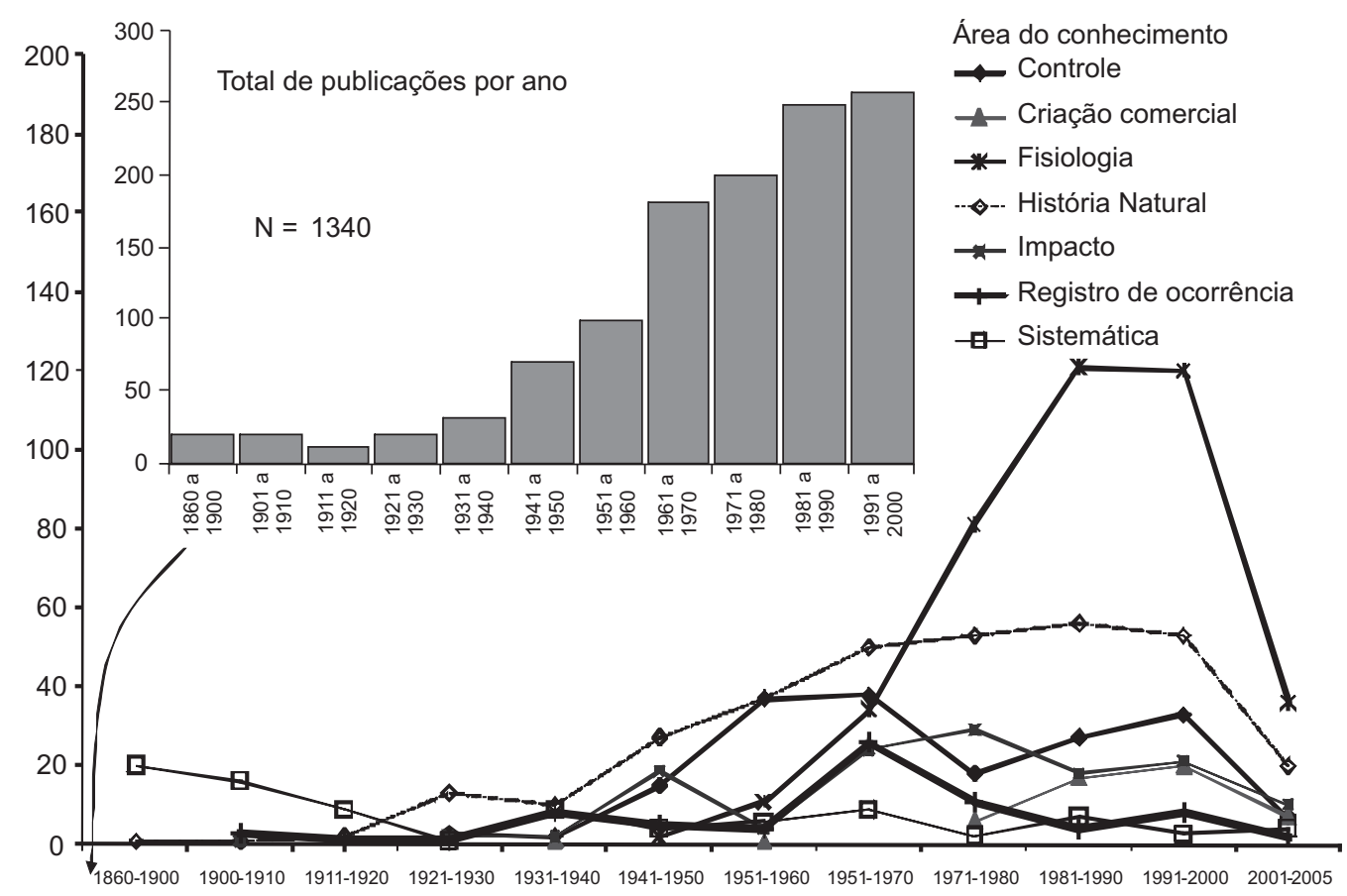

Figura 1. Total de publicações e número de publicações por área do conhecimento em cada década. 
normativas e trabalhos técnicos de manejo) do que empresas (14,1\% - comércio de conchas e criadores comerciais), páginas pessoais $(7,8 \%)$, ONGs $(7,5 \%)$ e mídia $(6,1 \%$ - jornais e revistas eletrônicas) $\left(\chi_{(6)}^{2}=575,4 ; \mathrm{p}<0,01\right)$. Os temas mais abordados foram o impacto $(29,6 \%)$, a criação comercial $(19,1 \%)$, a fisiologia $(17,6 \%)$ e outros $(15,5 \%$ - quando se tratava de outros animais e $A$. fulica era o assunto secundário), ocorrendo em menor quantidade informações sobre a história natural $(9,43 \%)$, manejo $(6,2 \%)$ e legislação $(2,3 \%)\left(\chi_{(6)}^{2}=222,46 ; \mathrm{p}<0,01\right)$. Os idiomas mais freqüentes foram o inglês $(64,5 \%)$ e o português $(19,3 \%)$, ocorrendo significativamente em menor freqüência outros idiomas (orientais: 7,6\%, alemão 4,1\%, francês 1,7\%, italiano $1,2 \%$, espanhol $1,1 \%$ e russo e indiano menos do que $1 \%)\left(\chi_{(9)}^{2}=2253,53 ; \mathrm{p}<0,01\right)$.

Dos 65 resumos publicados em anais de congresso, as informações sobre incentivo à criação (87\%) relacionadas à melhoria do rendimento de $A$. fulica na criação comercial, predominaram até 2000. A partir de 2001 a maioria dos estudos foi relacionada à biologia $(65,1 \%)$ e aos problemas do molusco como invasor $(39,5 \%)$.

\section{Impacto econômico}

Um ex-produtor de $A$. fulica revelou que as primeiras matrizes foram trazidas por ele na década de 1980 por iniciativa própria, sem nenhum apoio governamental. O caramujo apresentou excelente adaptabilidade, superior aos moluscos do gênero Helix Linnaues, 1758 (Helicidae). O início da atividade comercial se deu com a capacitação de criadores em potencial para abastecer a "indústria do escargot", através de um curso técnico, o qual era vendido durante feiras agropecuárias e incluía um kit com matrizes reprodutoras, caixas para o início da criação, rações e material didático teórico e áudio visual. Segundo o ex-produtor, o Brasil se tornou um dos principais países no ranking dos produtores dessa iguaria, sendo a produção nacional destinada ao mercado europeu. No entanto, na década de 1990, com a implantação do atual plano monetário, o negócio perdeu a lucratividade levando ao fim da atividade no Brasil. Os produtores ficaram sem alternativa de comercialização do produto, pois por questões culturais o mercado interno não supria a demanda, e assim a maioria dos produtores abandonou a atividade e liberou os caramujos no ambiente.

No Paraná a SESA-PR registrou a ocorrência de $A$. fulica em 132 dos 399 municípios do estado (Fig. 2). Entretanto, o impacto econômico do molusco não está evidente. A consulta aos órgãos gestores da agricultura do Paraná foi realizada a partir de oito contatos por correio eletrônico, com representantes da Empresa de Assistência Técnica e Extensão Rural (EMATER/ PR), Secretaria de Estado da Agricultura e Abastecimento do Paraná (SEAB/PR), Instituto Agronômico do Paraná (IAPAR) e Ministério da Agricultura, Pecuária e Abastecimento (MAPA/ $\mathrm{PR})$, resultando em apenas três respostas, das quais nenhuma apontou A. fulica como praga agrícola. O Serviço de Atendimento ao Cliente EMATER (SAC EMATER/PR) relatou que a empresa não detém informações sobre prejuízos agrícolas oca- sionados por caramujos, bem como o Departamento de Economia Rural (DERAL) vinculado a SEAB/PR. O IAPAR ressaltou que os prejuízos ocasionados por caramujos e lesmas sobre a agricultura paranaense são eventuais e de ocorrência local, não havendo estimativa sobre o grau do prejuízo.

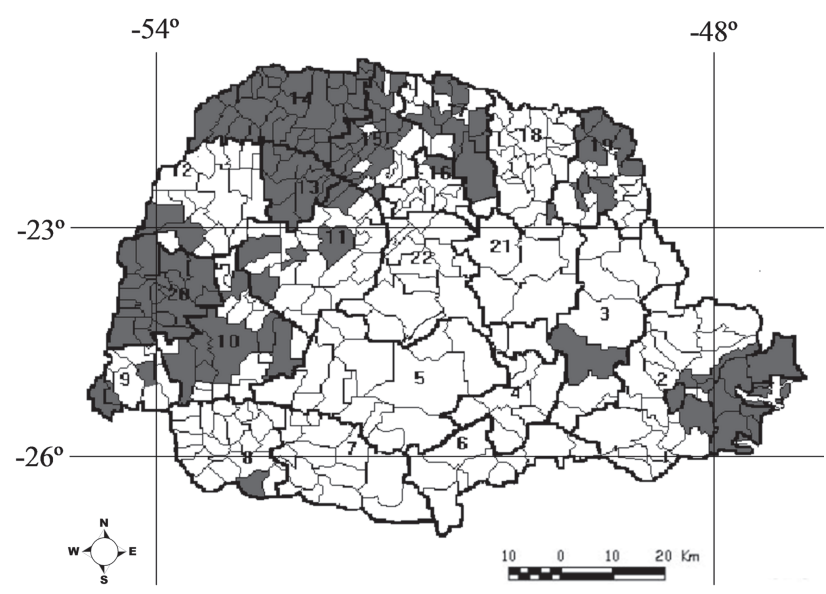

Figura 2. Mapa do estado do Paraná com os 132 municípios com ocorrência de A. fulica, destacados em escuro (período de 2000 a 2008). As divisas correspondem às 22 regionais de Saúde da SESA/ PR. Fonte: SESA/CSA/DV Zoonozes e intoxicações.

As entrevistas no município de Morretes também não revelaram a existência de grandes prejuízos econômicos. No entanto, as pessoas entrevistadas, na maioria homens (83\%), agricultores (54\%), com idade entre 20 e 40 anos (60\%) e residentes na área rural (60\%) disseram não cultivar horta devido à presença do caramujo exótico. Da mesma forma, em Guaraqueçaba, apenas $48 \%$ dos moradores ainda cultivavam horta e entre os que não cultivavam, a maioria $(58,3 \%)$ desistiu da prática após a chegada do caramujo invasor. Em Guaraqueçaba, todos os entrevistados afirmaram que $A$. fulica representa um transtorno pelo fato de comer as plantas do quintal, liberar uma grande quantidade de muco e fezes, além de representar uma ameaça à saúde. No entanto, a maioria dos entrevistados de Morretes (63\%) afirmou não estar sofrendo economicamente pela presença do invasor. O aumento da população de $A$. fulica foi relatado por $60 \%$ dos entrevistados, porém apenas metade destes faz algum tipo de controle. Segundo os relatos, 36\% disseram que o caramujo foi levado ao município por criadores comerciais, $27 \%$ através do transporte de material de construção e 3\% através do transporte da produção agrícola.

\section{Impacto em saúde pública}

No Encontro Brasileiro de Malacologia (2005) foram apresentados estudos com infecção de A. fulica por Angiostrongylus costaricensis (Morera \& Céspedes, 1971), Angiostrongylidae. No Brasil, as espécies de gastrópodes terrestres encontradas natural- 
mente infectadas por A. costaricensis são: Bradybaena similaris (Férussac, 1821), Bradybaenidae; Limax maximus (Linnaeus, 1758), Limacidae; Limax flavus (Linnaeus, 1758), Helix aspersa Muller, 1774, ambos Helicidae; Phyllocaulis variegatus (Semper, 1855, Veronicellidae; Phyllocaulis soleiformis (d'Orbigny, 1835), Veronicellidae; Sarasinula linguaeformis (Semper, 1885, Veronicellidae; Belocaulus angustipes (Heynemann, 1885), Veronicellidae; e Leptinaria unilamellata (d'Orbigny, 1835), Subulinidae (RAmbo et al. 1997, Laitano et al. 2001, Thiengo 2007). Embora estes registros sejam apenas para a região sul do país, casos da zoonose angiostrongilose abdominal também são registrados nas regiões sudeste e centro-oeste. O laboratório da Faculdade de Biociências e Instituto de Pesquisas Biomédicas da PUCRS em conjunto com a equipe de vigilância sanitária da Secretaria de Saúde de do Estado de Santa Catarina conduziu uma avaliação da potencialidade de $A$. fulica como transmissora de angistrongilíase. Os resultados revelaram que as larvas infectantes em $A$. fulica encontraram uma reduzida adaptação, inferior a $1 \%$, sendo menores do que os hospedeiros nativos. No entanto, THIENGo et al. (2005) acreditam que existe um risco em potencial de $A$. fulica participar do ciclo do nematóide, pois este apresenta baixa especificidade de hospedeiro, sendo que já existem registros de dois casos de meningite eosinofílica para o município de Cariacica, estado do Espírito Santo (CAldeira et al. 2007). Segundo Thiengo et al. (2005), a elevada carga parasitária do molusco evidencia a possibilidade de transmissão de outros tipos de enfermidades aos humanos, até então desconhecidas.

A SESA/PR considera a presença do molusco no Paraná um problema ambiental e agrícola e que em longo prazo pode se tornar um problema de saúde. Neste estado não há registro de doenças relacionadas a $A$. fulica e a análise de indivíduos de cinco regiões diferentes revelaram que nenhum estava infectado por vermes do gênero Angiostrongylus Kamenski, 1905. Os moradores do litoral paranaense temem a possibilidade de $A$. fulica causar problemas à saúde, porém, nenhum dos entrevistados soube relacionar os verdadeiros agravos de saúde ocasionados pelo caramujo invasor, sendo citado: doenças (33\%), meningite $(12,8 \%)$, queimaduras $(12,8 \%)$, problemas respiratórios $(7,7 \%)$, cegueira $(5,2 \%)$, barriga d'água $(5,2 \%)$, câncer $(5,2 \%)$, manchas de pele $(5,2 \%)$, coceiras $(5,2 \%)$, febre $(2,5 \%)$, feridas $(2,5 \%)$ e vômitos $(2,5 \%)$.

\section{Impacto ambiental}

Dos 44 pesquisadores consultados, apenas 36\% forneceram informações sobre $A$. fulica, sendo que apenas quatro deles ressaltaram o problema da invasão. Quanto ao impacto ambiental, as informações foram relativas ao registro do caramujo em florestas e remanescentes de florestas localizadas nos estados do Rio de Janeiro e do Paraná. O impacto foi relacionado ao consumo da vegetação nativa (sementes, brotos, folhas e frutos), competição por espaço e alimento com os moluscos nativos, principalmente de Megalobulimidae, além da captura e sacrifício da malacofauna nativa durante ações de controle. Outros dois fatores foram levantados na Reserva Bio- lógica Poços das Antas, Rio de Janeiro, como o consumo de $A$. fulica por espécies em risco de extinção como o mico leão dourado (Leontopithecus rosalia Lesson, 1840, Callithricidae) e as consequências prejudiciais desconhecidas que estes invasores podem trazer. O segundo fator é que a grande população do molusco exótico se torna recurso alimentar para espécies generalistas, que também podem aumentar suas populações.

No município de Morretes, 73\% dos entrevistados distinguiram $A$. fulica do caramujo nativo, enquanto que em Guaraqueçaba todas as pessoas conheciam A. fulica e $81 \%$ distinguiam do caramujo nativo. Dentre os entrevistados, $68 \%$ associaram a diminuição da população do caramujo nativo com a chegada do invasor.

As vistorias em campo conduzidas em 59 terrenos da área urbana do município de Guaraqueçaba resultaram na coleta de 1.877 caramujos e 1.630 conchas de $A$. fulica e sete caramujos e 15 conchas de indivíduos de Megalobulimidae. O número médio de $A$. fulica por terreno foi de 59,4 $\pm 71,9$ indivíduos (N $=59$, amplitude $=0-37$ ). O número de caramujos e conchas de indivíduos de Megalobulimidae coletados correspondeu a 0,6\% do total de $A$. fulica.

Na coleção malacológica do MHNCI estão registradas espécies de Strophocheilidae (22) e de Megalobulimidae (17), coletados desde 1953 até 1995 em diferentes regiões do Paraná, Brasil e América do Sul, mas não há exemplares de $A$. fulica. A coleção do MZUSP apresentou 902 Megalobulimidae, 290 Strophocholeidae e 27 A fulica relativos à ocorrência em todo território brasileiro.

\section{Avaliação da dispersão passiva e do potencial de recolonização}

Apenas em uma distribuidora de material de construção presente em Guaraqueçaba que abastece Guaraqueçaba e as ilhas da Baía de Paranaguá foram encontrados indivíduos de $A$. fulica no material que estava sendo transportado.

A taxa de recolonização de $A$. fulica nos 11 terrenos avaliados em Guaraqueçaba foi de 95,7\% em um período de cinco meses. Esta frequência foi o resultado da coleta total de 2.202 caramujos nas duas estações, não havendo diferença no número total de animais nas duas coletas (Fig. 3). No entanto, os caramujos registrados na primeira coleta $(5,1 \pm 1,8 \mathrm{~cm}, \mathrm{~N}=$ 1.126, amplitude $=0,5-15$ ) foram significativamente maiores do que os da segunda $(3,6 \pm 2,4 \mathrm{~cm}, \mathrm{~N}=1.077$, amplitude $=$ 0,5-12) (U = 363761, p < 0,001) (Fig. 3).

\section{Ações de controle}

As ações de controle de A. fulica no Brasil foram iniciadas a partir de 2001, com recomendações do Ministério da Agricultura e do Abastecimento (MAPA) para o controle do caramujo invasor apresentadas pela Sociedade Brasileira de Malacologia (Thiengo et al. 2007). Em 2002 foi elaborado o "Programa Nacional de Saneamento Ambiental da Invasão de Achatina fulica Preocupação Nacional" pelo Instituto Brasileiro de Helicicultura (IBH). Este programa objetivou capacitar profissionais de saú- 


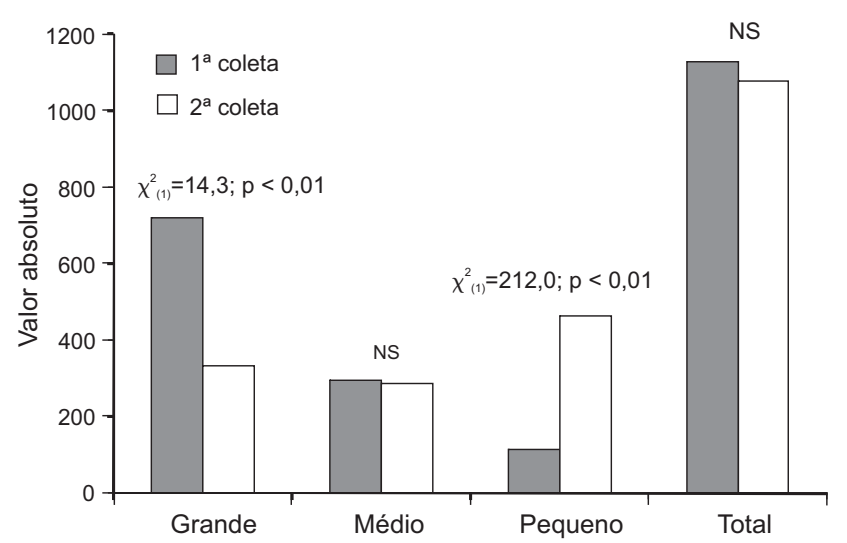

Figura 3. Número de indivíduos pequenos $(0,3-4,0 \mathrm{~cm}$ de comprimento de concha), médios $(4,1-6,0 \mathrm{~cm})$ e grandes (maiores que $6,0 \mathrm{~cm}$ ) de $A$. fulica coletados em Guaraqueçaba em duas amostragens seqüenciais. Os valores absolutos relativos à proporção de animais pequenos, médios e grandes foram comparados entre as duas amostragens através do teste do qui-quadrado, assim como o número total de animais amostrados, sendo as diferenças significativamente diferentes $(p<0,05)$ acompanhados pelo asterisco e não significativa por NS.

de, educação e líderes comunitários para implementação de ações do manejo em seus municípios. No entanto, a falta de continuidade não acarretou nos resultados esperados.

O Ibama/DF se mobilizou a partir de 2004 com do "Plano de Ação de Combate ao Caramujo Africano Achatina fulica Bowdich, 1822". Este projeto teve por objetivo prestar assessoria às prefeituras com a execução do "Dia C" ou "dia de combate ao caramujo africano". O "Dia C" se constitui de um feriado ou sábado aleatório em que o Ibama capacita funcionários públicos e estes atuam como agentes multiplicadores que supervisionam a coleta manual de caramujos e ovos de $A$. fulica executada por alunos das escolas locais. Esta ação, em geral, ocorre em apenas um dia, sem uma data ou frequência específica, mas com a intenção de serem continuadas pelas prefeituras (THIENGO et al. 2007).

A última medida aplicada pelo MMA, no ano de 2005, foi a elaboração do relatório das espécies exóticas invasoras registradas para o Brasil e dos ambientes e estados em que ocorrem em virtude do "Informe Nacional de Espécies Exóticas Invasoras". Estes projetos tiveram início em 2003, sendo conduzidos e coordenados pelo Instituto Hórus de Desenvolvimento e Conservação e pela The Nature Conservancy (TNC) (THIENGO et al. 2007).

\section{Legislação}

A legislação brasileira referente às espécies exóticas invasoras está presente no Decreto 4.339 de 22 de agosto de 2002, que instituiu a Política Nacional da Biodiversidade, descrevendo a regulamentação de medidas de controle, manejo e erradicação das espécies exóticas invasoras no Brasil. No en- tanto, para que possam ser conduzidas ações legais de combate é necessário estar ciente da forma como estas são consideradas pela legislação. A Lei de Fauna, 5.197 de 03 de janeiro de 1967 e a Lei dos Crimes Ambientais, 9.605, de 12 de fevereiro de 1998, definem que "a fauna silvestre se constitui de animais de espécies nativas, migratórias e quaisquer outras, aquáticas ou terrestres que tenham todo ou parte do seu ciclo de vida ocorrendo dentro do território brasileiro ou águas jurisdicionais brasileiras, sendo propriedade do Estado, estando proibida a sua utilização, perseguição, destruição, caça ou apanha". A definição de fauna silvestre apresentada por estas leis inclui espécies exóticas invasoras como A. fulica, embora em seus artigos subsequentes liberem a destruição de animais exóticos considerados nocivos à agricultura ou à saúde pública bem como a caça de animais domésticos asselvajados. Porém, estas ações esbarram na obrigatoriedade de uma licença expedida pela autoridade competente. No caso de $A$. fulica, outro empecilho para sua erradicação no Brasil, deve-se ao fato da espécie ser considerada apenas um potencial risco para agricultura e para a saúde humana. Segundo Thiengo et al. (2007) em 2003, o Ibama e o MAPA oficializaram a ilegalidade na comercialização de $A$. fulica e em 2004 uma lei similar foi promulgada no estado de São Paulo (Lei 11.756). A proibição da criação e comercialização em todo território nacional veio a partir de 2005 com uma instrução normativa número 73 do Ibama, a qual passou a considerar o caramujo-gigante-africano, A. fulica, como não pertencente à fauna silvestre nativa, sendo, portanto, uma espécie exótica invasora, nociva às espécies silvestres nativas, ao ambiente, à agricultura e à saúde pública, e autorizando a implementação de medidas de controle, coleta e eliminação. Tendo em vista que a responsabilidade sobre o manejo de $A$. fulica é dos estados e municípios, o Paraná por meio do Instituto Ambiental do Paraná (IAP) promulgou a Portaria número 74, de 19 de abril de 2007 apresentando a "Lista Oficial de Espécies Exóticas Invasoras para o Estado do Paraná". Esta lista inclui $A$. fulica e estabelece normas de controle para as EEI, designando ao IAP a responsabilidade sobre os procedimentos para licenciamento, monitoramento, fiscalização e controle de espécies exóticas invasoras.

\section{DISCUSSÃO}

O levantamento das publicações sobre $A$. fulica evidenciou que, apesar de haver informações, as mesmas são pouco acessíveis. Uma das dificuldades está relacionada ao fato de informações científicas sobre biologia básica, terem sido publicadas em revistas de pequena circulação e consequentemente de pouca difusão e pequena disponibilidade. A internet provavelmente é a primeira ferramenta usada para o levantamento de dados, sendo essencial tanto para o conhecimento científico quanto técnico e popular (BYERs et al. 2002). Porém, a predominância de referências científicas e em inglês pode dificultar a interpretação das informações pelos dois últimos. As informações existentes sobre $A$. fulica correspondem à problemática encontrada para 
outras espécies invasoras em um contexto global, revelando um conteúdo insuficiente em relação à invasão biológica, inacessível ou de difícil compreensão (BYERs et al. 2002). A dificuldade de acesso às informações sobre o caramujo invasor evidencia o despreparo para tratar dos problemas relacionados à sua introdução no país, tendo em vista que o conhecimento sobre a espécie é o primeiro passo para realizar ações de prevenção e de controle. Na tentativa de reverter essa situação o Ministério do Meio Ambiente estabeleceu como ação prioritária a divulgação pública de informações sobre espécies exóticas invasoras, incluindo A. fulica, através de estratégias que vão desde a inclusão do tema no currículo escolar até a criação de sites relacionados ao assunto (MMA 2005).

O fato da presente pesquisa não evidenciar a existência de impactos econômicos no Paraná pode ser devido à predominância da espécie invasora na área urbana (FIsCHER \& Colley 2004, 2005, SIMĨ̃o \& FISCHER 2004), causando principalmente danos em jardins e hortas. No entanto, A. fulica é reconhecida mundialmente como uma praga agrícola voraz, causando sérios prejuízos principalmente sobre culturas tropicais (PAIVA 2001, Vasconcellos \& Pile 2001, Raut \& Barker 2002, Teles et al. 2004), consumindo diariamente em torno de $10 \%$ do peso do seu corpo (Raut \& Barker 2002). Todavia, Mead (1979) afirma que $A$. fulica causa apenas danos locais sobre algumas espécies de vegetais e plantas ornamentais e considera que a comunidade científica e a mídia têm exagerado na divulgação do caramujo como praga. Segundo Mead (1979) e Civeyrel \& Simberloff (1996), durante o estabelecimento da população de $A$. fulica há uma grande infestação, mas que inevitavelmente entra em declínio e se extingue. No Brasil, $A$. fulica é considerada uma praga em potencial e os relatos sobre os prejuízos causados à produção agrícola estão relacionados apenas aos pequenos produtores (PAIVA 2001, Teles et al. 2004). Tendo em vista que o caramujo africano apresenta um longo histórico como praga agrícola em países tropicais e que no Brasil sua ocorrência atualmente é predominantemente nas áreas urbanas, causando prejuízos mínimos, o presente momento é fundamental para serem aplicadas medidas de controle e erradicação. Contudo, a falta de evidências de que $A$. fulica está causando danos no Brasil, diminui a preocupação relacionada aos prejuízos causados pelo caramujo exótico.

A baixa ocorrência de casos autóctones de angiostrongilíase no Brasil, como os dois registros de meningite eosinofílica em Cariacica, Espírito Santo (CALDEIRA et al. 2007) não descarta o risco eminente de saúde pública. Segundo Teles \& Fontes (1998), A. fulica é susceptível à infecção por Angiostrongylus spp. e muitos indivíduos coletados em vida livre no Brasil estão parasitados por outros nematóides. Vasconcellos \& Pile (2001) acreditam que o fato de não terem sido até então registradas $A$. fulica infectadas por Angiostrongylus spp. no Brasil, pode ser devido à realização de coletas pontuais e à ocorrência de variações sazonais do verme. No Brasil, o fato do caramujo invasor apresentar uma ampla distribuição geográfica eleva as chances de disseminação do nematóide por todo território brasileiro.
Entretanto, estudos recentes realizados por NeuHauss et al. (2007) no Brasil demonstram que $A$. fulica apresenta baixa susceptibilidade à infecção por Angiostrongylus spp., tanto A. costaricensis como de $A$. cantonensis. Porém, é importante ressaltar que, além da possível transmissão da angistrongilíase, deve se levar em consideração o fato de que o caramujo invasor pode ser vetor de outros parasitas que podem colocar em risco a saúde humana e de outros animais, como nematódeos que causam pneumonia em gatos (THIENGo et al. 2008). O potencial risco de transmissão de doenças causadas por $A$. fulica é o principal assunto relacionado ao caramujo invasor divulgado na mídia brasileira e também é a maior preocupação demonstrada pela sociedade.

$\mathrm{O}$ impacto ambiental causado pela invasão de $A$. fulica tem sido o principal problema apontado em estudos anteriores realizados no Paraná (Fischer \& Colley 2004, 2005) e recentemente foi registrada a invasão do caramujo em ambiente natural (Fischer et al. 2006). A principal ameaça, inclusive em ambiente antrópico, tem sido relacionada aos danos causados aos moluscos nativos pertencentes a Megalobulimidae. Segundo Fischer \& Colley (2004), as pessoas não distinguem a espécie invasora das nativas e acabam sacrificando os caramujos nativos juntamente com o invasor. A utilização de métodos de controle químico irregulares também tem agravado o impacto sobre a fauna nativa. Possivelmente, os impactos ambientais ainda estejam se iniciando. Segundo RAUT \& BARKER (2002), a invasão de $A$. fulica em áreas naturais apresenta um processo inicial de herbivoria das plantas nativas, seguido pela alteração do sistema ecológico natural, supressão das espécies de moluscos nativos (competição por espaço, como: sítio de repouso e postura de ovos; e por alimento) e por fim, contribui para o estabelecimento de outras espécies invasoras. No entanto, os danos ambientais são difíceis de serem mensurados, pois esta avaliação exige uma comparação entre as condições ambientais antes e depois da chegada da espécie invasora.

Os resultados obtidos na consulta às coleções científicas evidenciaram que esse recurso não é possível de ser usado para subsidiar resolução do problema de espécies invasoras. Segundo Byers et al. (2002), a consulta às coleções científicas deveria servir para diagnosticar a fase entre a dispersão e o impacto de uma espécie invasora, principalmente aquelas já estabelecidas. No entanto, como não há depósitos regulares em coleções isto se torna inviável. A dificuldade para diferenciar a espécie invasora das nativas é acentuada uma vez que alguns panfletos informativos sobre $A$. fulica apresentam ilustrações de uma espécie de Achatina Lamarck, 1799 diferente da introduzida no Brasil. Outro fator que dificulta a identificação é o fato de $A$. fulica apresentar um forte polimorfismo durante o desenvolvimento ontogenético.

A ampla distribuição de $A$. fulica no Brasil tem sido favorecida por diferentes estratégias de dispersão, entre as quais está o transporte passivo. No presente estudo, o caramujo invasor foi observado sendo transportado aderido ao material de construção, ressaltando as observações de AlicATA (1966) quan- 
to à habilidade de $A$. fulica se dispersar através de diferentes substratos. O elevado potencial de recolonização de $A$. fulica em Guaraqueçaba corrobora com outra avaliação realizada por SImĩ̃o \& FIsCher (2004) no município de Pontal do Paraná. Estes estudos indicaram que a efetividade da coleta manual deve focar estratégias de ação contínua, evitando a re-colonização da população do caramujo que pode voltar com mais vigor, após os animais mais velhos serem retirados do ambiente.

As ações de controle e manejo de $A$. fulica levantadas neste estudo não têm contribuído para a diminuição de suas populações. Segundo Byers et al. (2002), o controle de espécies exóticas deve focar o melhor local para aplicar a ação e envolver uma estratégia de detecção precoce, erradicação, monitoramento e controle para evitar outras invasões. ZILLER (2004) ressalta que as tecnologias para combater as espécies exóticas invasoras ainda são rudimentares. Esta afirmação é corroborada por estudos que apontam a coleta manual de animais e ovos como uma das principais alternativas de controle para A. fulica, embora seja a medida mais trabalhosa e cara, e em alguns casos pouco eficiente (Byers et al. 2002, CowIE \& Robinson 2003, Ziller 2003). O sucesso no controle e erradicação de $A$. fulica após o seu estabelecimento é raro. Um exemplo de sucesso ocorreu em Miami, EUA, em que uma população em fase inicial de estabelecimento composta por 18.000 indivíduos foi erradicada após seis anos de trabalho, envolvendo coleta manual por profissionais capacitados, iscas com veneno e extensiva campanha educativa com um investimento de aproximadamente um milhão de dólares (Cowie \& Robinson 2003, Simberloff 2003). Cowie \& Robinson (2003) apresentam uma série de medidas para controle de moluscos invasores destacando a quarentena e ressaltando que essa medida de vigilância oficial preveniu que $A$. fulica se tornasse problema nos EUA, Austrália e Ilhas Fiji tanto por prevenir a instalação quando por promover a erradicação rapidamente. Levando em consideração que a ocorrência de $A$. fulica no Paraná é caracterizada por populações recentes e estabelecidas em áreas urbanas. Somado ao fato que as estimativas para o tamanho das populações estão em torno de 403.000 para Pontal do Paraná (SIMĨ̃o \& Fischer 2004) e 53.000 para Guaraqueçaba (FIscher \& Colley 2004), a demora na execução de ações de manejo tornarão praticamente impossível o controle e a erradicação da espécie. Esta hipótese é reforçada pelos estudos de Fischer et al. (2006) que registraram o estabelecimento da população de $A$. fulica na Floresta Atlântica, sugerindo a saturação do ambiente antrópico e alertando para a rápida infestação de novas áreas naturais que poderiam causar irreversíveis impactos ambientais.

A legislação brasileira e o conhecimento sobre espécies exóticas invasoras no país estão em fase de amadurecimento. Segundo Ziller (2003), é preciso haver uma comunhão de interesses entre legislação vigente, análise de risco e desenvolvimento institucional e técnico no país. Só assim, será possível encontrar soluções para o uso sustentável e conservação dos recursos naturais. A experiência de países desenvolvidos mos- tra que a legislação adequada deve ser clara, aplicável e abranger não apenas os aspectos ambientais, mas o setor produtivo, a sociedade, a comunidade cientifica e os órgãos gestores, estabelecendo para cada um, seus direitos e deveres.

A aquisição de informações sobre $A$. fulica, mesmo se tratando de uma espécie bem conhecida e reconhecida como invasora há mais de um século em diferentes países, deixou clara a dificuldade enfrentada para o manejo de espécies invasoras no Brasil. O passo inicial de alguém que se depara com uma suposta espécie invasora é procurar informações sobre ela, porém estas devem ser de fácil acesso e compreensão. A dificuldade na identificação dos animais e o acesso às informações precisas sobre seus reais impactos atrasam o início do controle, que obviamente seria mais efetivo antes do estabelecimento da espécie invasora. Assim, a falta de esclarecimentos conduz a sociedade a se conformar com a presença do caramujo invasor. O presente estudo reforça que a integração entre o conhecimento acadêmico, os órgãos gestores e a sociedade é extremamente necessária para que seja possível o manejo e o controle de espécies invasoras. A ação de controle deve ser adaptada a cada realidade, focada no diagnóstico prévio e em uma metodologia que envolva a divulgação de informações claras e a instrução da sociedade, além de um monitoramento contínuo. E, por fim, deve-se considerar que a situação de $A$. fulica é apenas um termômetro da necessidade de adequação de uma série de procedimentos que visem o controle de espécies com potenciais impactos ambientais, econômicos e de saúde pública.

\section{AGRADECIMENTOS}

A Pró-Reitoria Comunitária e de Pesquisa da PUCPR; Gisélia Rúbio (SESA/PR), Mônica S. Simião (CEM/UFPR); Luiz R. Simone (MZUSP), Fábio Faraco (IBAMA) e Willian do Amaral (IBH) pelas comunicações pessoais. Aos pesquisadores que participaram das entrevistas, bem como aos moradores do Município de Guaraqueçaba e Morretes. A Ferenc Polena pela concessão da entrevista. Aos membros discentes do NEC, PUCPR pela colaboração durante a fase de coleta: Nicole M. Latoski, Izabel S. Nering, Ana Paula Winter, Fernando Leivas, Areli Zortea, Alessandra B. da Silva, Anderson Scorsato, Adriana Machado e Lygia Hasselmann, Marcia Cziulike e Samira Schahad.

\section{LITERATURA CITADA}

AlicATA, J.E. 1966. The presence of Angiostrongylus cantonensis in islands of the Indian Ocean and probable role of the giant African snail, Achatina fulica, in dispersal of the parasite to the Pacific islands. Canadian Journal of Malacology 41: 1041-1047.

Byers, J.E.; S. Reichard; J.M. Randall; I.M. Parker; C.S. Smith; W.M. Londale; A.E. Atrinson; T.R. Seastedt; M. Williamson; E. Chornesky \& D. Hayes. 2002. Directing research to reduce the impacts of nonindigigenous species. Conservation Biology 16: 630-640. 
Caldeira, R.L.; C.L.G.F. Mendonça; C.O. Goveia; H.L. Lenzi; C. Graeff-Teixeira; W.S. Lima; E.M. Mota; I.L. Pecora; A.M.Z. Medeiros \& O.S. Carvalho. 2007. First record of molluscs naturally infected with Angiostrongylus cantonensis (Chen, 1935) (Nematoda: Metastrongylidae) in Brazil. Memórias do Instituto Oswaldo Cruz 102: 887-889.

Civeyrel, L. \& D. Simberloff. 1996. A tale of two snails: is the cure worse than the disease? Biodiversity and Conservation 5: 1231-1252.

Cowie, R.H. \& D.G. Robinson. 2003. Pathways of introduction of noindigenous land and freshwater snails and slug, p. 93122. In: G. RUIZ \& J.T. CARLTON (Eds). Invasive species: vectors and management strategies. Washington, Island Press, 484p.

Fischer, M.L. \& E. Colley. 2004. Diagnóstico da ocorrência do caramujo gigante africano Achatina fulica Bowdich, 1822 na APA de Guaraqueçaba. Estudos de Biologia 26 (54): 4350.

Fischer, M.L. \& E. Colley. 2005. Espécies invasoras em reservas naturais: caracterização da população de Achatina fulica Bowdich, 1822 na Ilha Rasa, Guaraqueçaba, Paraná. Biota Neotropica 5 (1): 1-17. Available online at: http://www. biotaneotropica.org.br/v5n1/pt/abstract?article+BN0330501 2005 [Accessed: 25/VIII/2008].

Fischer, M.L.; M.S. Simião; E. Colley; R.D. Zenni; D.A.T. Silva \& N. LATOSKI. 2006. O Caramujo exótico invasor na vegetação nativa em Morretes, PR: diagnóstico da população de Achatina fulica Bowdich, 1822 em um fragmento de Floresta Ombrófila Densa Aluvial. Biota Neotrópica 6 (2). Available online at: http://www.biotaneotropica.org.br/ v6n2/pt/abstract?short-communication+bn03306022006 [Accessed:20/I/2008].

Lowe, S.; M. Browne \& S. Boudjelas. 2004. 100 of the world's worst invasive alien species. A selection from the global invasive species database. Available online at: www.issg.org/database [Accessed: 20/I/2008].

Laitano, A.C.; J.P. Genro; R. Fontoura; S.S.L. Branco; R.L. Maurer; C. Graeff-Teixeira; J.M. Milanez; L.A. Chiaradia \& J.W. Thomé. 2001. Report on the ocurrence of Angiostrongylus costaricensis in southern Brazil, in a new intermediate host from the genus Sarasinula (Veronicellidae, Gastropoda). Revista da Sociedade Brasileira de Medicina Tropical 34: 95-97.

MEAD, A.R. 1979. Economic malacology with particular reference to Achatina fulica, p. 1-150. In: V. Fretter \& J. Peaks (Eds). Pulmonate. London, Academic Press, 417p.

Mма 2005. I Simpósio brasileiro sobre espécies exóticas invasoras. Brasília, Ministério do Meio Ambiente. Available online at: http://www.mma.gov.br/invasoras [Accessed: 20/ I/2008].

Neuhauss, E.; M. Fitarelli; J. Romanzini \& C. Graeff-Teixeira. 2007. Low susceptibility of Achatina fulica from Brazil to infection with Angistrongylus costaricensis and A. cantonensis. Memórias do Instituto Oswaldo Cruz 102: 49-52.
PAIVA, C.L. 2001. Achatina fulica (Moluscos) nova praga agrícola e ameaça á saúde pública no Brasil. Fontes de informação impressas e digitais. Centro de Memória UNICAMP (GEHT/CMU). Available online at: http://www.geocities. com/lagopaiva/achat_tr.htm [Accessed: 20/I/2008].

Rambo, P.R; A.A. Agostini \& C. Graeff-Teixeira. 1997. Abdominal Angiostrongylosis in Southern Brazil - Prevalence and Parasitic Burden in Mollusc Intermediate Hosts from Eighteen Endemic Foci. Memórias do Instituto Oswaldo Cruz 92: 9-14.

Raut, K. \& G. Barker. 2002. Achatina fulica Bowdich and others Achatinidae pest in tropical agriculture, p. 55-114. In: G. BARKer (Ed.). Mollusks as croup pest. London, UK. CAB Publishing, 400p.

Simberloff, D. 2003. How much information on population biology is needed to manage introduced species? Conservation Biology 17: 83-92.

Simĩ̃o, M.S. \& M.L. Fischer. 2004. Estimativa e inferência do método de controle do molusco exótico Achatina fulica Bowdich, 1822 (Stylommatophora; Achatinidae) em Pontal do Paraná, litoral do Estado do Paraná. Cadernos de Biodiversidade 4: 74-83.

Teles, H.M.S. \& L.R. Fontes. 1998. Angiostrongilíase e escargot: nova ameaça a saúde pública. Cadernos de Saúde 30: 2426.

Teles, H.M.S. \& L.R. Fontes. 2002. Implicações da introdução e dispersão de Achatina fulica Bowdich, 1822 no Brasil. Boletim do Instituto Adolfo Lutz 12: 3-5.

Teles, H.M.S.; L.R. Fontes \& W. Amaral. 2004. Pesquisa nacional de opinião pública sobre a espécie do caramujo Achatina fulica. Instituto Brasileiro de Helicicultura, Fundação CEDIC 1-24. Available online at: http:// www.cedic.org.br/Bio/pesquisa.pdf [Accessed:20/I/2008].

Thiengo, S.C. 2007. Helmintoses de interesse médico-veterinário transmitidas por moluscos no Brasil, p. 287-294. In: S.B. Santos; A.D. Pimenta; S.C. Thiengo; M.A. Fernandez; R.S. AbSAlão (Eds). Tópicos em Malacologia Ecos do XVIII Encontro Brasileiro de Malacologia. Rio de Janeiro, Ed. Corbã, $406 \mathrm{p}$.

Thiengo, S.C.; A.F. Barbosa; P.M. Coelho \& M.A. Fernandez. 2005. Moluscos exóticos com importância médica no Brasil. Brasília, I Simpósio Brasileiro Sobre Espécies Exóticas Invasoras. Available online at: http://www.mma.gov.br/ invasoras[Accessed: 20/I/2008].

Thiengo, S.C.; F.A. Faraco; N.C. Salgado; R.H. Cowie \& M.A. FERnANDEz. 2007. Rapid spread of an invasive snail in South America: the giant African snail, Achatina fulica, in Brazil. Biological Invasions 4: 1-10.

Thiengo, S.A.R. C.; M.A. Fernadez; E.J.L. Torres; P.M. Coelho \& R.M LANFREDI. 2008. First record of a nemetode Metastrongyloidea (Aelurostrongylus abstrusus larvae) in Achatina (Lissachatina) fulica (Mollusca, Achatinidae) in Brazil. Journal of Invertebrate Pathology 98: 34-39. 
Vasconcellos, M.C. \& E. Pile. 2001. Ocorrência de Achatina fulica no Vale do Paraíba, Estado do Rio de Janeiro, Brasil. Revista Saúde Pública 35: 582-584.

ZILLER, S.R. 2003. Contaminação biológica: Conceitos, contexto e prática, p. 186-198. In: A. BARGER (Ed.) Áreas protegidas: conservação no âmbito do cone sul. Pelotas, PUC, 223p. ZiLler, S.R. 2004. Espécies exóticas e invasoras em unidades de conservação. In: Anais do IV Congresso Brasileiro de Unidades de Conservação. Fundação O Boticário de Proteção a Natureza 2: 74-77.

Submitted: 08.XII.2008; Accepted: 26.XI.2009.

Editorial responsibility: Heraldo Vasconcelos 